\title{
Releasing the Brakes
}

Dear Reader,

Participants from last year's “ $3^{\text {rd }}$ Agile Automotive Conference” seemed to arrive at the somewhat pessimistic consensus that, "Agility won't work in many enterprises until a few members of their executive boards opt for retirement." Prof.

Dr. Hans-Christian Reuss addresses this sentiment in his commentary on page 74 . "A responsible and disciplined development team cannot work with a sufficient degree of agility unless it has the trust of the management and unless trust prevails among its members," says the Director of the Chair in Automotive Mechatronics at the University of Stuttgart.

It is also crucial for executives to begin showing trust, especially with respect to those with leadership skills, business acumen and the courage to implement transformative processes based on good business models. Although Dr. Stefan Sommer met those very criteria, the former CEO at ZF was nonetheless unable to persuade the automotive supplier's majority shareholder, the city-owned Zeppelin Foundation, to accept his proposals for restructuring the enterprise. Sommer and one of his supporters, the former Supervisory Board Chairman Giorgio Behr, decided to leave the enterprise at the end of 2017.

Conservative corporate cultures hinder innovative employees. In general, priority is given to meeting the expectations of shareholders and owners by paying out dividends on an annual basis. In contrast, investments that are sustainable and profitable in the long term, including those that go beyond securing existing market shares to the conquering of new markets, tend to be put on hold. The rules of the game are essentially left uncontested, including by those who praise the courage of agile start-ups while being quick to point out their failure to turn a quick profit. "The enormous amount of money that will need to be spent all at once on electric vehicles, digitization and driverless technology is daunting," one market observer noted. This has led to a decision-making logjam at the level of the top management. Start-ups building anew with the help of less risk-averse and more patient investors and entirely free of restructuring tasks can proceed with greater agility. And that is what is giving rise to new brands of electric vehicles.

Yes, engineers employed by the major automobile manufacturers have begun to demand flexibility and agility. They have begun to participate in new teams and to attempt to persuade the top management to embrace more efficient methods of cooperation. But for at least two reasons, they have grounds for despair. First, they continue to fall on deaf ears. Second, agility initiatives wind up suffocating in the decision-making logjams.

In any case, it is not only enterprises that are called upon to act more quickly. Lawmakers, municipalities and government agencies have also done their part to discourage innovation. More forthright efforts on the part of these decision makers could awaken the curiosity of conservative investors.

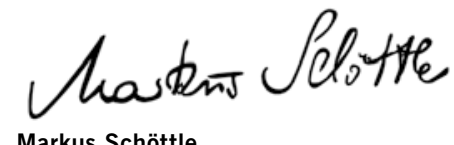

Markus Schöttle

Deputy Editor in Chief

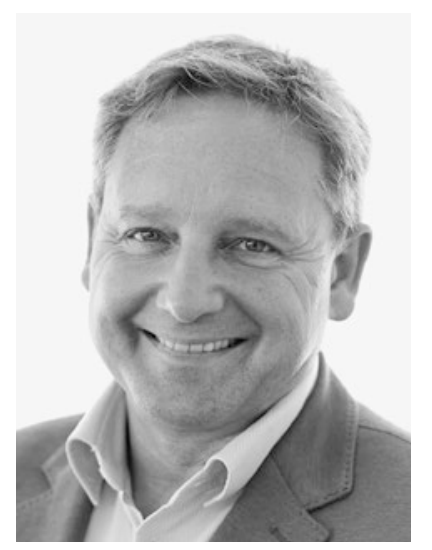

